\title{
Erroneous Data Transfer
}

National Cancer Institute

\section{Source}

National Cancer Institute. Erroneous Data Transfer. NCI Thesaurus. Code C139515.

The device software fails to transfer the expected data within a system or to another device. 